\title{
Modelling of photonic wire Bragg gratings
}

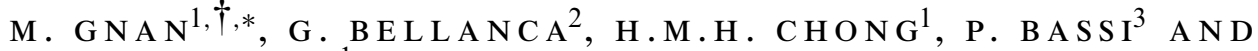 \\ R.M. DE LA RUE ${ }^{1}$ \\ ${ }^{1}$ Optoelectronics Research Group, Department of Electronics and Electrical Engineering, The University, \\ Glasgow, G12 8QQ, Scotland, UK \\ ${ }^{2}$ Dipartimento di Ingegneria, University of Ferrara, Via Saragat 1, I 44100 Ferrara, Italy \\ ${ }^{3}$ Dipartimento di Elettronica Informatica e Sistemistica, University of Bologna, Viale Risorgimento 2, \\ I 40136 Bologna, Italy \\ $\dagger$ present address: Department of Electronics \& Electrical Engineering Rankine Building, Oakfield Avenue, \\ Glasgow, G12 8LT UK \\ (*author for correspondence: E-mail: m.gnan@elec.gla.ac.uk)
}

Received 30 September 2005; accepted 19 January 2006

\begin{abstract}
Some important properties of photonic wire Bragg grating structures have been investigated. The design, obtained as a generalisation of the full-width gap grating, has been modelled using 3D finite-difference time-domain simulations. Different types of stop-band have been observed. The impact of the grating geometry on the lowest order (longest wavelength) stop-band has been investigated - and has identified deeply indented configurations where reduction of the stop-bandwidth and of the reflectivity occurred. Our computational results have been substantially validated by an experimental demonstration of the fundamental stop-band of photonic wire Bragg gratings fabricated on silicon-on-insulator material. The accuracy of two distinct 2D computational models based on the effective index method has also been studied - because of their inherently much greater rapidity and consequent utility for approximate initial designs. A 2D plan-view model has been found to reproduce a large part of the essential features of the spectral response of full 3D models.
\end{abstract}

Key words: finite-difference time-domain, optical waveguide modelling, photonic crystal, photonic wire, silicon on insulator, waveguide grating

\section{Introduction}

In recent years the race for faster optical communication and data processing, whether for switching or computation, has motivated research towards devices with more functional elements directly interconnected on a single chip - or even, with full processing on the chip, to truly integrated optical circuits (Noda et al. 2002; Almeida et al. 2004; Kimerling et al. 2004).

For these objectives, planar photonic crystal (PhC) based devices have been recognised as providing a possible implementation, since they offer enhanced possibilities as compared with devices based on total internal reflection (TIR). These possibilities come from the inherent complexity of 2D PhC band-structure and the additional effects of various types of defect engineering. However, because their characteristics are ultimately produced by multiple diffraction effects, $\mathrm{PhCs}$ have drawbacks in terms of fabrication 
complexity and significant propagation losses, unless the fabrication precision and quality are of the highest possible standard (Van Thourhout et al. 2005).

On the other hand, devices based on TIR, such as photonic wires ( $\mathrm{PhWs}$ ) have demonstrated various elements such as $90^{\circ}$ abrupt bends, T-junctions (Espinola et al. 2001; Ahmad et al. 2002) and Mach-Zehnder structures (Zhao et al. 1995). For wavelength selective functionalities, photonic wires with periodic variation of the waveguide-width and/or depth can be employed (Krauss et al. 1997; Foresi et al. 1997; Lalanne and Hugonin 2003; Jugessur et al. 2004). Essentially two kinds of design for the modulation of the effective refractive index have been described: the 'on-off' design, (intuitively similar to a 1D periodic structure and exemplified by side-wall gratings or full-width gap gratings), and the periodic insertion of holes, (which can be considered as incorporating a form of 1D or 2D photonic crystal (Joannopoulos et al. 1995)). For all of these structures, silicon-on-insulator (SOI) provides an attractive choice of base material, even potentially for devices requiring active functionality (Van Thourhout et al. 2005). Photonic wire structures have been employed to realise microcavities with distributed Bragg reflector mirrors that can exhibit high reflectivity and small size, because the refractive index can be strongly modulated (between semiconductor and air values) in the central region of the waveguide, where the field is highest. On the other hand, photonic wire grating structures are intuitively more prone to diffraction losses. For these reasons, a design that enables an adjustable and more controllable reflectivity is potentially important. Consideration of a waveguide with a periodically varied width (a generalisation of the full-width gap grating) provides the necessary base for designing the structures required. Moreover, from a fabrication point of view, the photonic wire Bragg grating makes for more homogeneous dry-etch processing, since the ions employed are readily able to escape from the interaction region, instead of being trapped in the $\mathrm{PhC}$ holes.

Proper design of these $\mathrm{PhW}$ Bragg grating structures requires preliminary modelling to account for the many degrees of freedom allowed by this structure - and we shall describe here a study on the properties of the $\mathrm{PhW}$ Bragg grating. Starting from a photonic wire realised in silicon-oninsulator material, the grating is obtained as a periodic insertion of recesses into the wire. In the following sections a three dimensional model is investigated via the finite-difference time-domain (FDTD) method. The properties of the Bragg grating transmission spectrum will be investigated and the dependence of its reflection properties on the geometrical parameters will be analysed. Experimental results for the transmission stop-band behaviour of fabricated structures will then be given to validate the theoretical results. Finally, the accuracy of two distinct $2 \mathrm{D}$ reduced computational models will 
(a)

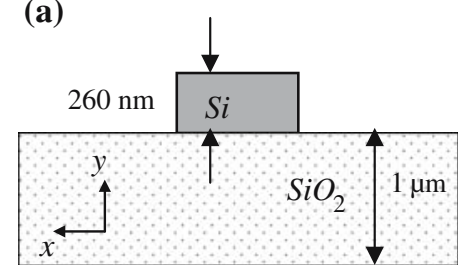

(b)

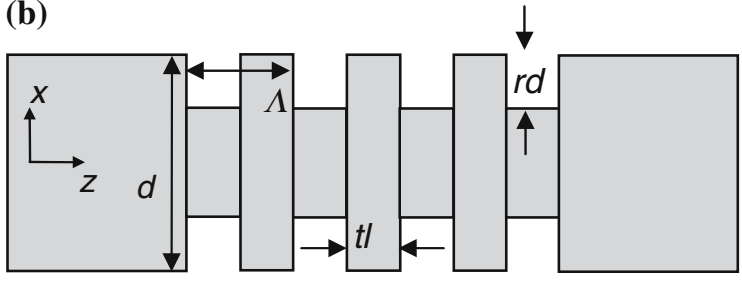

Fig. 1. Schematic of the cross-section of the photonic wire (a), and of the top view of the grating (b).

be studied, in order to find modelling tools that will enable the rapid realisation of approximate initial designs.

\section{Design description}

Considering SOI material with a $260 \mathrm{~nm}$ thick silicon (Si) core layer - and a $1 \mu \mathrm{m}$ thick silica $\left(\mathrm{SiO}_{2}\right)$ lower cladding layer, a $500 \mathrm{~nm}$ wide photonic wire waveguide may be defined by a complete vertical etch through the core, as shown schematically in Fig. 1a. This waveguide is intended to be used at wavelengths around $1.52 \mu \mathrm{m}$, at which the refractive index of the materials are 3.479 and 1.445, for $\mathrm{Si}$ and $\mathrm{SiO}_{2}$ respectively (Palik 1998). The width $d$ has been fixed at $500 \mathrm{~nm}$, because considering the polarisation (quasi-TE, with the main electric-field component parallel to the $x$-direction), and the wavelength of operation, a waveguide with this width supports only a single even transverse mode of propagation. As the device has perfect transverse symmetry, this choice ensures operation in a mono-mode regime that, it has been shown, can be maintained in conjunction with low propagation losses in the fabricated waveguide (Van Thourhout et al. 2005).

The indented grating structure was obtained by the periodic insertion of recesses on both sides. The description of the grating is defined by the parameters: grating period $(\Lambda)$, recess depth $(r d)$, tooth length $(t l)$, as shown in Fig. 1b. In the following discussion, the behaviour of the cascade of (input) wire, grating, (output) wire (as exemplified in Fig. 1b) will be presented.

\section{3D model}

In order to describe the behaviour of the device, its spectral response was calculated. For the simulation of the behaviour of the device, 3D-FDTD code was employed (Taflove 1995). The method, which relies on the discretisation of Maxwell's equations, provides the description of the time 
evolution of the electromagnetic field without any assumption about the number and the characteristics of the propagating modes. For a review of other algorithms capable of performing a similar analysis, the references in (Čtyroký et al. 2002) should be consulted. In that paper, in a deeplyetched, 1D-periodic, grating geometry, a high level of agreement between the FDTD method and other numerical techniques has been demonstrated.

The cascade of input wire, grating, output wire was reproduced by a mesh of $\delta x=\delta y=20 \mathrm{~nm}$ and $\delta z=19.5 \mathrm{~nm}$. The simulation domain was terminated by uniaxial perfectly matched layers (UPML). The excitation section was set by the input wire at distance of $2 \mu \mathrm{m}$ from the beginning of the grating, together with an output port computing reflected field. Symmetrically, the output port computing transmitted field was set on the output wire. The source employed was a sinusoid enveloped by a Gaussian pulse, with a transverse profile corresponding to the fundamental mode of the input wire waveguide: by Fast-Fourier Transforming the $E_{x}$ field-component sampled at the output ports, the frequency response of the device under investigation was obtained, with just a single simulation run. To guarantee a frequency resolution of about $20 \mathrm{GHz}$, the simulations were run for 46000 time steps. To speed-up the computations, a parallel FDTD code was used on a cluster of 8 Personal Computers (Bellanca et al. 1999).

In the following description, firstly the transmission and loss spectra of a generic configuration are shown and analysed with the help of the field distribution. The loss spectrum $(L)$ is defined as that part of the power which, not being detected, is assumed to be scattered by the device - and is calculated as $L=I-(T+R)$, where $I$ is the input power that is launched in the waveguide in the fundamental mode (refer to the appendix for a more detailed explanation). Then the influence of the variation of the geometry will be explained.

When dealing with distributed reflectors, it is evident that the amount of reflected power depends strongly on the number of periods. In the following results, unless explicitly stated, the length of the grating is 16 periods: this allows a good compromise between computational load and the visibility of the basic effects. The stop-band width is conveniently defined here as the distance between the peaks of the Bragg ripples, and the centre wavelength is then defined as their arithmetic mean wavelength.

Figure 2 shows the transmission and loss spectra of a generic configuration obtained for $\Lambda=390 \mathrm{~nm}, r d=120 \mathrm{~nm}, t l=\Lambda / 2$. It is possible to distinguish various features in the spectrum - and these features will be analysed in detail in the following discussion.

The first order stop-band is centred at $\lambda_{0}=1528 \mathrm{~nm}$ and has a width $\Delta \lambda=93 \mathrm{~nm}$. Within the stop-band the loss curve has a monotonic trend: in particular the longer wavelengths show low total loss and the shorter wavelengths show higher loss. Plots of the field configuration of the Bloch mode 

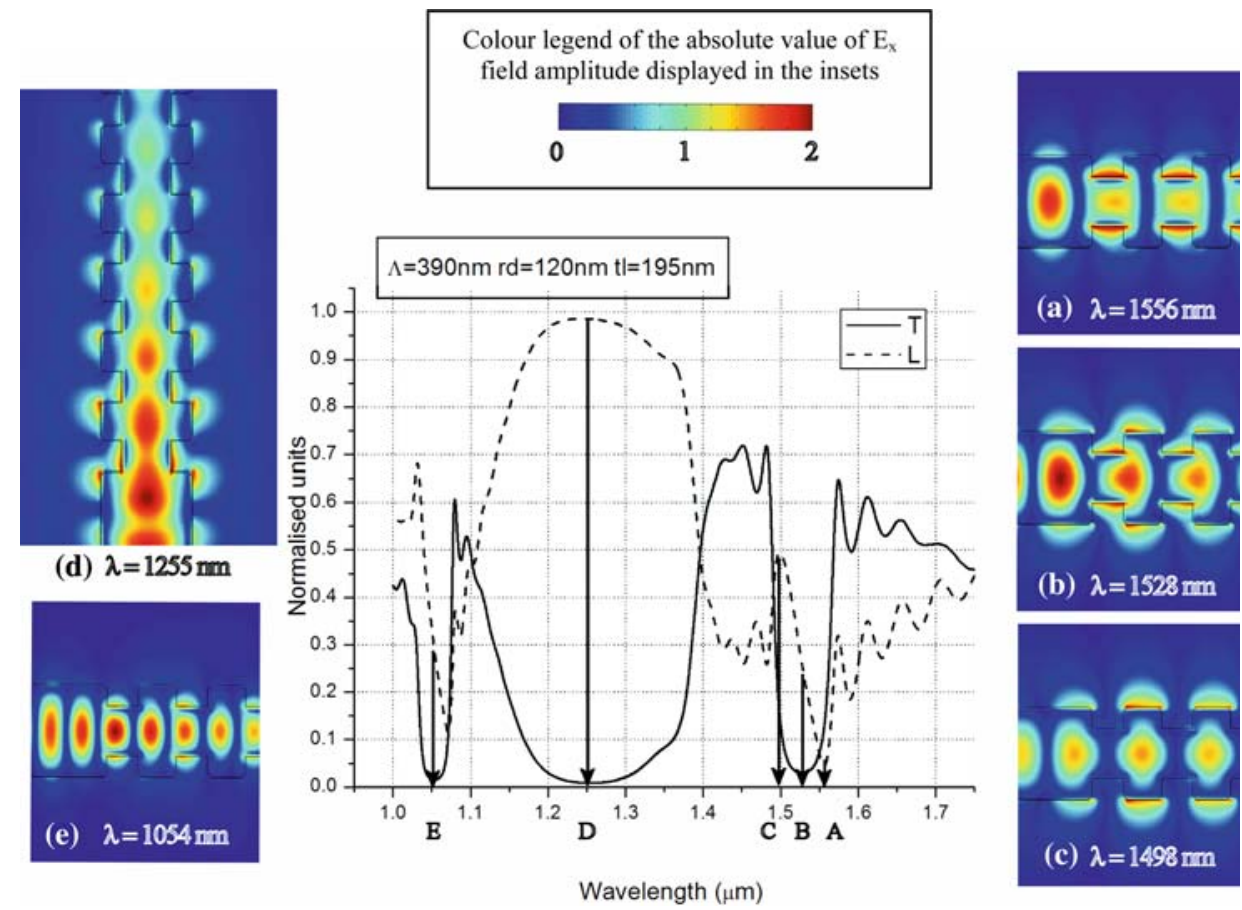

(b) $\lambda=1528 \mathrm{~mm}$

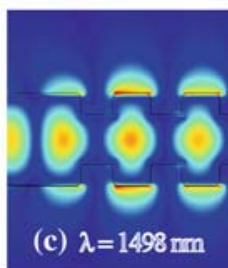

Fig. 2. Transmission $(T)$ and loss $(L)$ spectra of a generic 3D configuration $(\Lambda=390 \mathrm{~nm} r d=120 \mathrm{~nm}$ $t l=195 \mathrm{~nm}$ ). For some wavelengths within the stop-bands the plots of $E_{x}$ field absolute value are shown. A full period is given by the distance between two maxima of the displayed absolute value.

supported by the grating at the loss minimum $(\lambda=1556 \mathrm{~nm})$, at the middle point of the stop-band - and at the maximum loss point $(\lambda=1498 \mathrm{~nm})$ show that, within the stop-band $(\lambda=1528 \mathrm{~nm})$, the stationary wave progressively undergoes shifts in the position of its field maxima (which have a full period of $2 \Lambda$ ) from the wider sections of the grating, to the shorter ones.

As the field becomes more spread along the (transverse) $x$-direction, its mismatch with the incoming photonic wire mode increases. As a result, the field is increasingly scattered at the interface between the two, as is depicted in the figures. Further simulations on this device were performed with a doubled length (32 periods) - and showed that the average loss within the stop-band increases by $0.9 \%$. These considerations allow the conclusion that, within the stop-band, the grating Bloch mode is confined, and the loss of power is due to the mismatch at the two interfaces between the grating section and the unstructured photonic wire at its input and output. Similar effects were reported in the case of planar photonic crystal geometries (Sauvan et al. 2005). Further demonstration that the modes of the wires waveguides and grating sections are confined is given by the absence of leakage of power into the substrate: a simulation performed in the worst 
case ( $r d=250 \mathrm{~nm}$, i.e. a fully recessed grating profile), with a lower silica cladding of doubled thickness (in 16 periods), shows a maximum variation in the transmission as low as $0.1 \%$ over a bandwidth spanning from $\lambda=1$ to $\lambda=2 \mu \mathrm{m}$. A second transmission stop-band is centred at $\lambda_{0}=1262 \mathrm{~nm}$ and has the width $\Delta \lambda=334 \mathrm{~nm}$. Within this stop-band, the losses tend to reach unity - and, in this case, the field distribution shows coupling to radiative modes. A third transmission stop-band is centred at $\lambda_{0}=1054 \mathrm{~nm}$ and has the width $\Delta \lambda=53 \mathrm{~nm}$. Considering the behaviour of the losses and the field distribution (the periodicity of the maxima is here $\Lambda$ ), it is evident that this is a higher order stop-band of the first order one centred at $\lambda_{0}=1528 \mathrm{~nm}$. This kind of repetition of stop-bands then goes further as the wavelength decreases.

As a partial conclusion, this grating configuration shows two kinds of stop-bands, one in which confined modes are coupled - and one in which radiative modes are coupled. For practical applications, the first order stopband can be used as a reflector, since the confined Bloch modes can be combined with the mono-mode operating regime of the $\mathrm{PhW}$ waveguide.

The behaviour of this stop-band is therefore investigated further for its dependence on variations in the geometry of the grating. To do so, a simplified model of the interaction that occurs within the grating will be used. The aim is to provide a better vision of the properties of the grating itself - without the effect of the input wire waveguide, which so far has been understood as generating scattering losses at the interfaces. Supposing this to be the only effect of the wire outside the grating (Palamaru and Lalanne 2001), a simplified model is described in the appendix - and yields the reflectance of the grating itself. According to the model and considering unitary input power $(I=1)$, the transmission in that case would be $T_{G}=T /(1-L)$, and the reflection would be $R_{G}=1-T_{G}$.

For the analysis of the geometrical variations, $\mathrm{rd}$ and $\mathrm{tl}$ will be the free parameters. The grating period $\Lambda$ is kept constant for the sake of simplicity: it is assumed, as a first approximation, to be only a scaling parameter, the effect of which would result mainly in shifting the spectral features, with reduced distortion. This assumption is justified from basic photonic crystal theory (Joannopoulos et al. 1995). Furthermore, the width of the wire $(d)$ is also considered as fixed, since it is designed for operation at $1.52 \mu \mathrm{m}$. As for photonic crystal structures, the filling factor $(f f)$ can be defined as the ratio of the air fraction in a period - and is then given by $f f=2 r d(\Lambda-t l) /(d \cdot \Lambda)$.

Figure 3 shows a generic representation of the evolution of the characteristics of the first order stop-band, respectively as a function of $r d$ (a) and $t l$ (b). Common features can be found: with increasing filling factor, according to the reduction of the average index value per period, the position of the stop-band (in the figure the stop-band edges are shown as filled circles) 
(a)

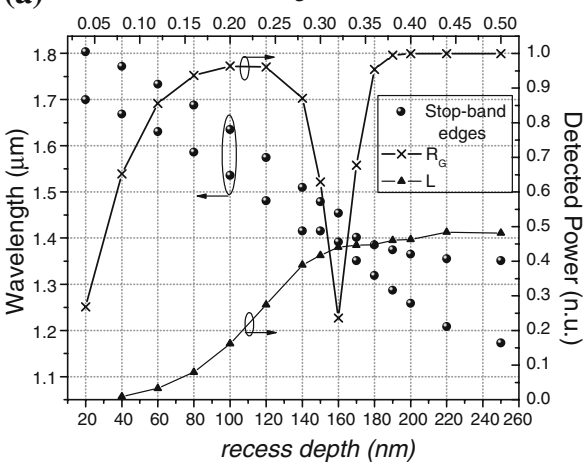

(b) filling factor

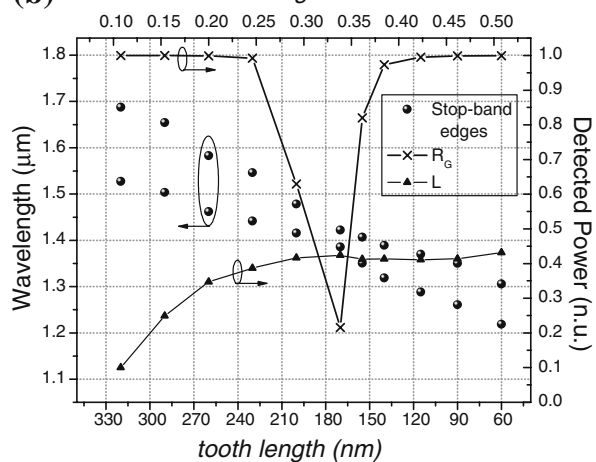

Fig. 3. First order stop-band behaviour according to variation of: a) recess depth $(\Lambda=390 \mathrm{~nm}$, $t l=195 \mathrm{~nm})$, and $\mathrm{b})$ tooth length $(\Lambda=390 \mathrm{~nm}, r d=150 \mathrm{~nm})$. The values have been calculated using the simplified grating model.

shifts to shorter wavelengths. On the contrary the width slowly varies and becomes larger for the highest values of both $r d$ and $t l$. The average loss in the stop-band (triangles) increases with the filling factor, showing the increasing mismatch between the Bloch mode of the grating and the mode of the wire waveguide. In the figures, the reflectance of the grating (considered as the maximum reflectance value within the stop-band), according to the approximate model $\left(R_{\mathrm{G}}\right)$, is shown (the crosses represent calculated points and the connecting lines are mainly as an aid to visualisation). In the regions around $r d=160 \mathrm{~nm}$ and $t l=170 \mathrm{~nm}$ the geometrical configuration makes the multiple reflections in each period of the grating become progressively less destructive, thus enhancing transmission, as is clearly visible by a drop in the reflectance and a reduction in the width of the stop-band.

It can be concluded that, even when the corrugation of the grating is as small as $r d=100 \mathrm{~nm}$, the 16-period long grating can operate with high reflectivity. On the other hand, losses quickly become large as the filling factor increases, showing the need for an engineered interface with the unstructured wire waveguide in a manner similar to that used in refs (Akahane et al. 2005; Englund et al. 2005; Sauvan et al. 2005). Finally, the presence of geometries in which the interplay of multiple reflections enhances transmission has been evidenced.

\section{Experimental results}

In order to obtain validation of the theoretical results, fabrication of the gratings was performed using electron-beam lithography and dry-etching 

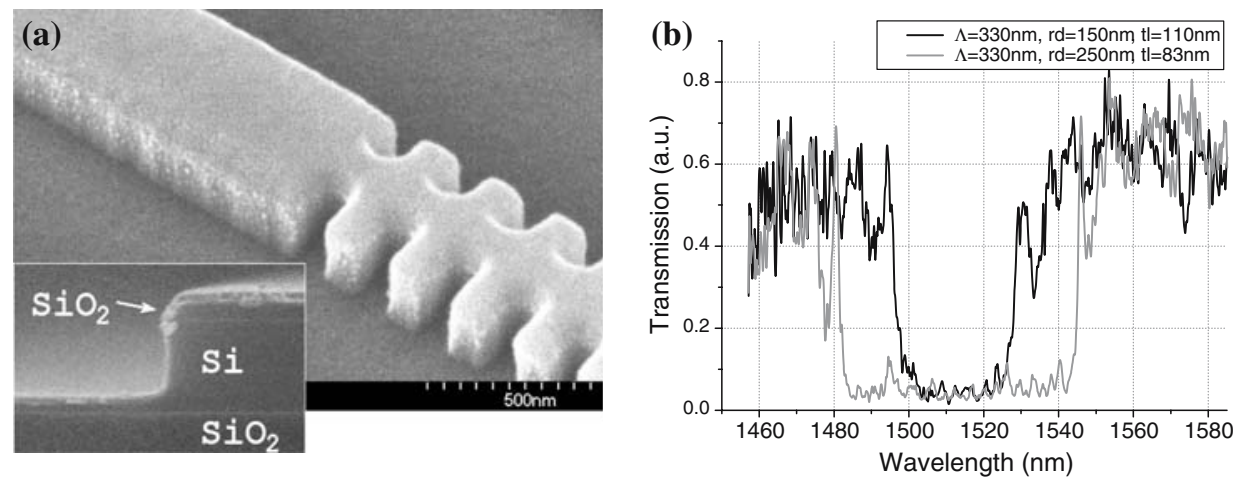

Fig. 4. (a) Scanning electron microscope micrograph of a PhW Bragg grating fabricated on SOI; the inset shows the section of the wire: it is possible to recognise the silicon core not completely etched through, and the layer of silica left on top of the wire. (b) Measured transmission of the fabricated $\mathrm{PhW}$ Bragg gratings showing stop-band in the $1.52 \mu \mathrm{m}$ spectral region: the stop-band position and width vary depending on the geometrical parameters which are specified in the legend.

technologies. As will be described in detail in a future publication, because of the actual details of the fabrication processes, the cross-sectional geometry of the photonic wires and grating sections was different from those in the $3 \mathrm{D}$ computational model described above. As shown in the micrograph in Fig. 4, a silica layer with an estimated thickness of $35 \mathrm{~nm}$ was left on top of the silicon core and, furthermore, the etching of the silicon was incomplete - and left $\sim 60 \mathrm{~nm}$ of silicon on top of the lower cladding. Even though the fabricated structure was different from the simulations, it showed a clear stop-band, as shown in Fig. 4, which gives the experimental transmission results. To obtain these results TE polarised light was end-firecoupled into and out of the waveguides, and the output light was detected using a germanium photodiode.

The two curves for the measurements show that, even if the fabricated device deviates from the perfection of the original model, there is, in each case, a stop-band that is determined by its parameters. Obviously, the absolute values of stop-bandwidth and position are different from those already shown, since the actual device is different. Nevertheless the measurement gives a positive indication for the probable existence of the other stopbands predicted by the computational simulation.

As a partial conclusion, the characteristic effect of the grating is strong enough to be clearly apparent, even when the dimensions realised drift away from the initial design. But the behaviour of the grating exhibits sensitivity to the position and width of the stop-band. Furthermore, our work demonstrates the intrinsic limits of the computational modelling process: whenever agreement with measurements is sought, the model has to be adapted to the actual dimensions of the fabricated structure. In practice 
obtaining agreement has to become an iterative sequence obtained after many repetitions of the fabrication processes and adaptation of the model. In that sense, time becomes an important parameter, since there may need to be several cyclic repetitions of the simulation and fabrication processes. Therefore, since approximation is unavoidable, the most rapid computations, even if quite approximate, should be used, at least in the early stages of the design process. We shall therefore move to investigations of whether it is possible to study this kind of structure with a reduced computational model. In particular, we shall study a 2D computational model based on the effective index method, which is a widely diffused and much used general approach.

\section{Reduced models}

The effective index method is one of the most common ways to reduce the dimensionality from $3 \mathrm{D}$ to $2 \mathrm{D}$. The basic concept for this method is to replace the $3 \mathrm{D}$ representation of the waveguide by an equivalent $2 \mathrm{D}$ waveguide, in which the core is represented by the effective refractive index of a slab guide that is obtained by considering the remaining dimension of the problem. This dimension is characteristically the waveguide thickness, along a direction perpendicular to the plane of the substrate surface. In the case of a waveguide with a rectangular cross-section, the task is to solve the electromagnetic problem of a reduced structure, thus leading to approximation errors that are greater in absolute magnitude for the case of strong confinement. The method was originally proposed by Knox and Toulios as a simplified quasi-analytical means to model waveguides in 1970 (Knox and Toulios 1970). Since then the effective index method has been used many times and many improvements within the basic approach have been proposed (Chiang 1996; Han et al. 2003).

Figure 5 shows the three 'natural' cross-sections for 2D analysis of the structure, considering the directions of the three axes that provide a reference for the analysis. Since part (a) corresponds to a cross-section of the waveguide and does not provide any information on the grating part, the plan view (part (b)) and the axial cross section (part (c)) can be used as 2D approximations of the structure. For the two resulting modelling problems, an analysis similar to the one already shown for the 3D model has been performed and the transmission spectrum of a generic configuration has been obtained, followed by further investigations of the behaviour of the fundamental order stop-band according to variations of the geometry. In order to allow for a simple comparison with the $3 \mathrm{D}$ data, the electromagnetic simulation still used the FDTD method- but now with two characteristic dimensions. The issue of accuracy deserves a remark: as the approach 


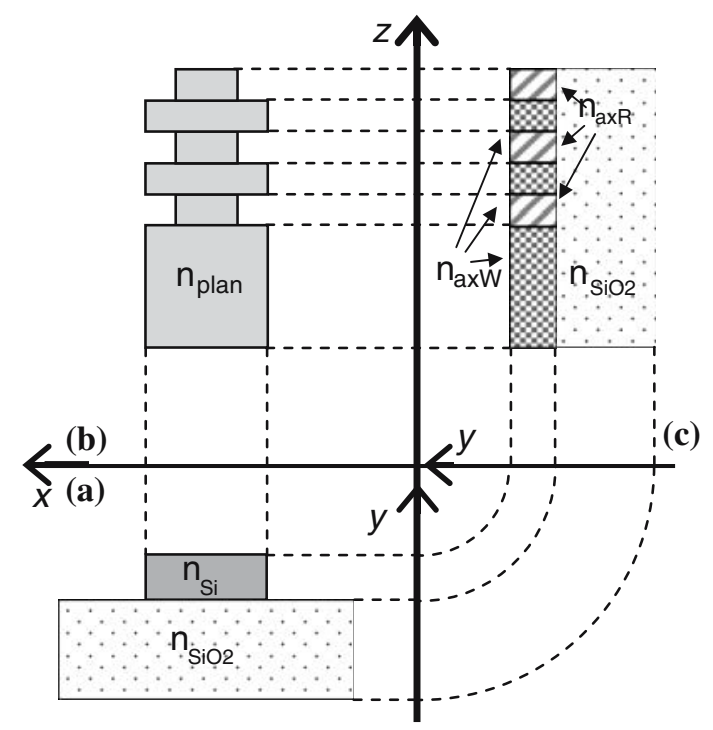

Fig. 5. Three possible orthogonal cross-sections of the PhW Bragg grating: (a) waveguide cross section, (b) plan view, (c) axial cross section.

used here has been to find a simple model that can approximate the structure, and the 2D approach is inherently prone to approximation errors, there is little point in using a very accurate mesh, which implies that the emphasis can be placed on reducing the computation time. The error introduced by using a relatively coarse mesh in the $2 \mathrm{D}$ computation can in fact be considered as contained within the 2D approximation itself - and could be reduced by an iterative optimisation stage.

\subsection{D PLAN-VIEW MODEL}

In the 2D plan-view model, a $2 \mathrm{D}$ simulation of the propagation in the $z x$-plane is performed with a structure obtained from the projection on to that plane (Fig. 5b). The device is then modulated using a refractive index $\left(n_{\text {plan }}\right)$ that is the value for the slab waveguide obtained by considering the distribution in the y-direction (the vertical direction), with regions defined as: (i) an infinite upper-cladding of air, (ii) a $260 \mathrm{~nm}$ thick silicon core and (iii) an infinite silica lower cladding, as already stated. (The actual silica lower cladding thickness of $1 \mu \mathrm{m}$ is thick enough for this purpose). As before the central wavelength of operation is $\lambda=1.52 \mu \mathrm{m}$, which gives an effective refractive index value of 2.97. Laterally the index is considered to be 1 . 


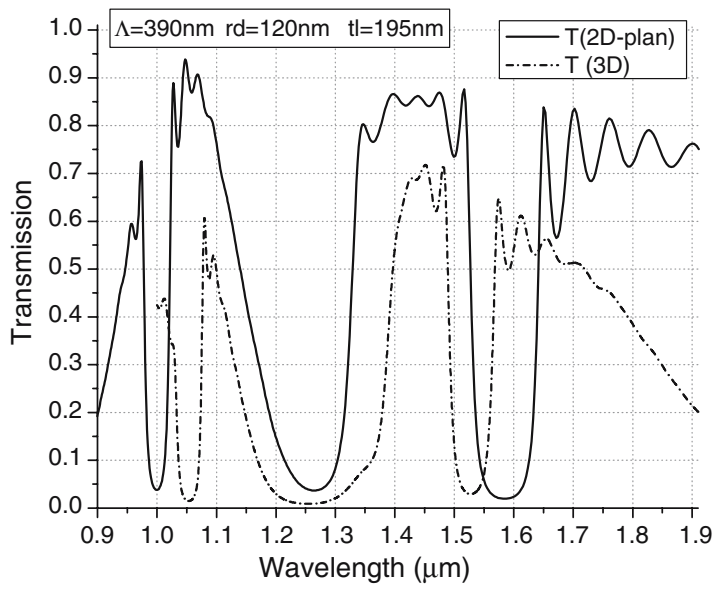

Fig. 6. Comparison of transmission of 2D plan view model (continuous line) and full 3D model (dashed line).
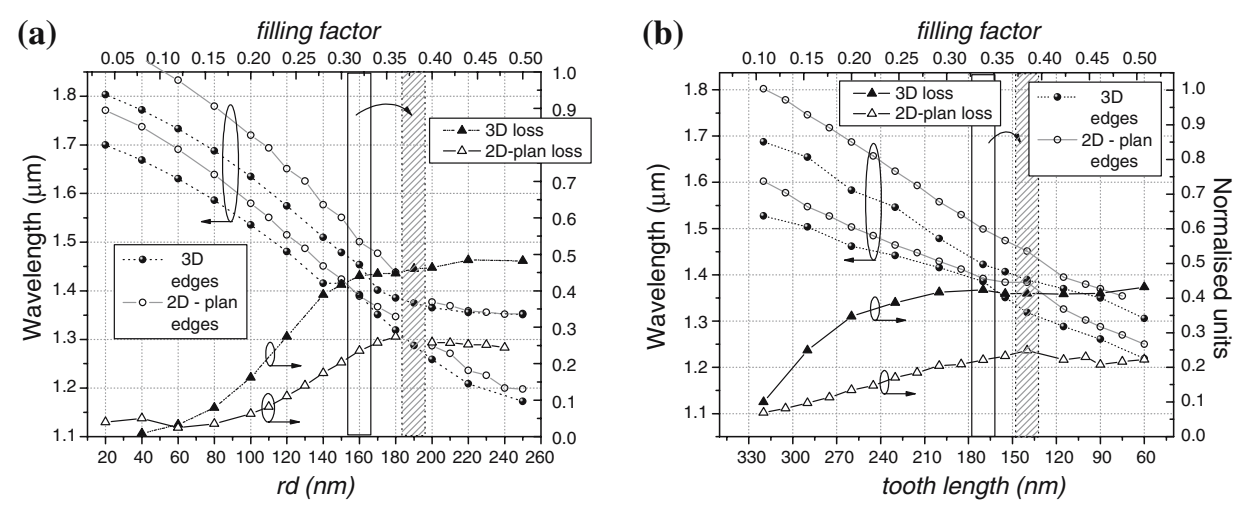

Fig. 7. Comparison of the properties of the first order stop-band according to $3 \mathrm{D}$ (filled symbols) and 2D (empty symbols) plan view models. The geometry is varied by: (a) recess depth $(\Lambda=390 \mathrm{~nm}$, $t l=195 \mathrm{~nm})$, and (b) tooth length $(\Lambda=390 \mathrm{~nm}, r d=150 \mathrm{~nm})$. The values have been calculated using the simplified grating model. The empty and shaded rectangles represent the regions of reduced reflectivity for the 3D and 2D-plan view models respectively.

Considering the same geometry analysed in section 3 for the $3 \mathrm{D}$ case (Fig. 2) - Fig. 6 shows the transmission spectra for the 2D plan-view model superimposed on the $3 \mathrm{D}$ case. It is possible to distinguish all the stop-bands of the $3 \mathrm{D}$ computation replicated in the $2 \mathrm{D}$ one, but with some distortion, as well as translation of the stop-band and other feature positions. This relative behaviour is expected quite simply because the effective refractive index determined by the waveguide dimension is a strong function of wavelength - while we have taken it to be constant. Specifically for the lower order stop-band, this drift compared to the $3 \mathrm{D}$ values is quantified as $3.6 \%$ 
( $57 \mathrm{~nm})$ for the stop-band centre $\lambda_{0}$, and $44 \%(41 \mathrm{~nm})$ for the stop-bandwidth $\Delta \lambda$. There are several ways in which the relative behaviour between the $2 \mathrm{D}$ and $3 \mathrm{D}$ simulations can be compared. In Fig. 7 we have considered the properties of the lowest order stop-band according to the variation of $r d$ (a), and $t l(\mathrm{~b})$, with $\Lambda$ and $d$ fixed at the same values shown for Fig. 3. As for the $3 \mathrm{D}$ case, the calculated values correspond to the simplified model described in the appendix. The figure shows that the stop-band is always shifted with a distortion that reduces for higher values of filling factor - and that the losses are reduced (by almost one half) in the 2D case. Moreover, as for the 3D case, the behaviour of the maximum reflectivity exhibits a region of reduced values (exemplified by shaded rectangles) corresponding to $r d \sim 190 \mathrm{~nm}$ and $t l \sim 140 \mathrm{~nm}$. In that case, the drift from the $3 \mathrm{D}$ values (exemplified by empty rectangles) is $18.7 \%$ for $r d$ and $17.6 \%$ for $t l$.

\subsection{D AXIAL CROSS SECTION ANALYSIS}

In the 2D axial cross-section model (Fig. 5c), a 2D simulation of the propagation in the $z y$-plane is performed with a structure obtained from the projection on to that plane. The device is then modulated using a refractive index that is the value for the slab waveguide obtained by considering the distribution in the $x$-direction. The structure is more complex than in the case of the plan-view as the index distribution (considered along the $x$-direction) varies more strongly in the $z y$-plane. The area corresponding to the projection of the wire is assigned a refractive index $\left(n_{\mathrm{axW}}\right)$ that is the effective refractive index of a slab consisting of a $500 \mathrm{~nm}(=d)$ thick silicon layer in air. The recessed regions of the wire have a refractive index $\left(n_{\mathrm{axR}}\right)$ equal to that of a similar slab with a core thickness reduced to $d-2 r d$ - and the silica cladding and air regions preserve their refractive index.

Considering the same geometry analysed in section 3 for the 3D case (Fig. 2) - Fig. 8a shows the transmission spectra for the 2D axial cross-section model superimposed on the $3 \mathrm{D}$ one. In the curve for the two-dimensional model, it is possible to distinguish two major stop-bands, around $\lambda=1.7 \mu \mathrm{m}$ and $\lambda=0.9 \mu \mathrm{m}$, that are shifted and roughly three times as wide as the corresponding ones in the $3 \mathrm{D}$ model. Moreover, considering variations of the geometry (i.e. of the value of $n_{\mathrm{axR}}$ in Fig. $5 \mathrm{c}$ ), the situation does not improve, since the stop-bandwidth difference remains high (Fig. 8b) - and the geometries that lead to reduced reflectivity cannot be modelled.

As a conclusion about the 2D modelling approach, considerable differences of behaviour between the two models have been evidenced, which 
(a)

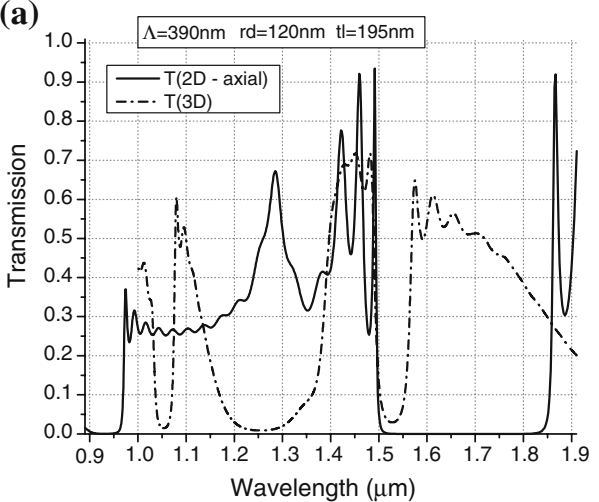

(b) $-\Lambda=390 \mathrm{~nm}$ rd $=120 \mathrm{~nm} \mathrm{t}=195 \mathrm{~nm}$ (2D axial)

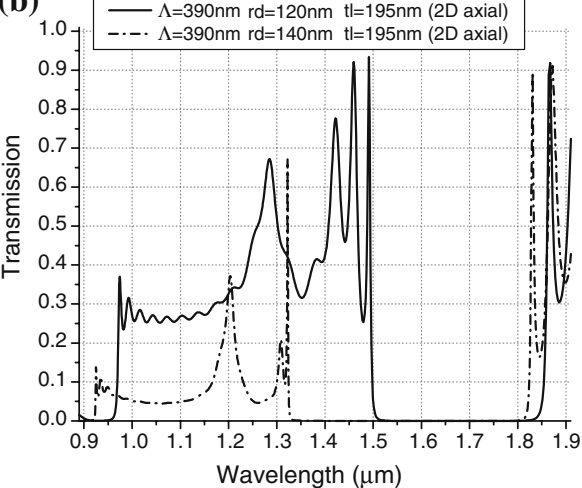

Fig. 8. (a) Comparison between transmission of 2D axial cross-section and full 3D models. (b) Transmission spectra according to two different values of $r d$.

can be attributed to the different properties of the sets of planar modes created by the two equivalent geometries - and to the levels of approximation introduced by the effective index method (which is related to the order in which the cross-sections of the waveguide are considered (Chiang 1996)).

The 2D plan-view model appears to reflect the full 3D model much more closely than the 2D axial-cross section one. In fact it reproduces the major features of the whole spectrum and, with respect to the lower order stopband, it maintains a similar level of accuracy when the geometry varies, even reproducing the reductions of reflection. However, the overall deviation of the $2 \mathrm{D}$ plan-view model from the $3 \mathrm{D}$ model was not negligible (3.6\% of the 3D stop-band centre wavelength, and $44 \%$ of the $3 \mathrm{D}$ stopbandwidth, for our example).

Even if these discrepancies are considered to be quite large, an iterated optimisation of the model to account for factors such as fabrication process drifts (for example an optimisation that uses different equivalent refractive indexes or that adjusts dimensions in the equivalent geometries) may improve the results for the $2 \mathrm{D}$ model bringing errors down to values which can be considered tolerable for an initial design stage.

\section{Conclusions}

The properties of photonic wire Bragg grating structures have been studied. Their design, obtained as a generalisation of the full-width gap grating, has been modelled and simulated - using 3D FDTD algorithm. Various kinds of stop-band have been identified, with the lowest order band being investigated in detail and showing the dependence of its width, position and 


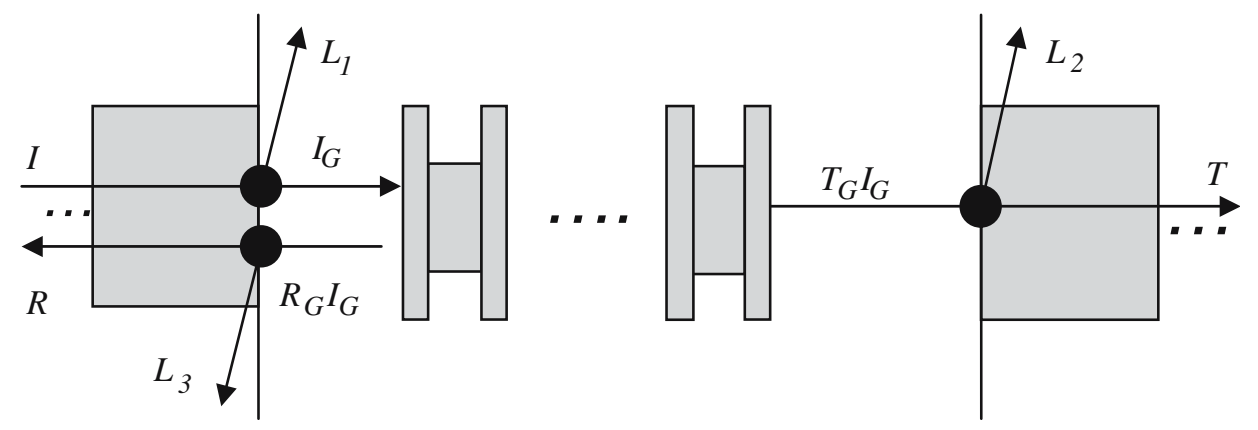

Fig. 9. Schematic of the power flow within the PhW Bragg grating operating as a reflector.

maximum reflectivity according to the geometry. Even for small corrugation levels, the reflectivity is large - thus enabling short reflector structures, while the existence of geometries for which the interplay of multiple reflections enhances transmission has been demonstrated. The main source of scattering has been identified as being the mismatch between the mode of the wire and the Bloch mode of the grating. These results have been validated by experimental demonstration of measured stop-bands in $\mathrm{PhW}$ Bragg gratings fabricated on SOI material. Finally, in order to find modelling tools that enable the rapid realisation of approximate initial designs, the accuracy of two distinct 2D reduced computational models based on the effective index method has been studied. Our results show that only a 2D plan-view model is required for initial approximated studies, since it reproduces all the essential features found in the spectra of full 3D models.

\section{Acknowledgements}

The authors thank Davide Castaldini for valid help in setting-up the FDTD code. Marco Gnan and Richard M. De La Rue acknowledge the financial support of the EPSRC (UK) through the Ultrafast Photonics Collaboration (UPC). Harold M.H. Chong acknowledges the financial support of the EPSRC (UK). Part of this research is supported by the Ministero dell'Istruzione dell'Università e della Ricerca (MIUR), Italy, and by the University of Ferrara under the project Nano \& Nano.

\section{Appendix}

In order to isolate the behaviour of the PhW Bragg grating when used as a reflector, a calculation similar to the transfer matrix formalism can 
be performed. The whole structure is divided into three blocks: the input wire, the grating and the output wire. They sustain guided and radiative modes for which the coupling is evaluated at their interfaces through overlap integrals (correlation) (Bienstman and Baets 2001). Figure 9 exemplifies the structure and the following power flow description: the guided input power $(I)$ is converted from a wire mode into a forward propagating Bloch mode, causing some coupling to radiative modes (i.e., losses, $L_{1}$ ); the forward and backward Bloch modes couple together without losses, and the power which reaches the end of the grating is attenuated by a factor $T_{\mathrm{G}}$, which stands as the transmittance of the isolated grating. The mode conversion into the output wire waveguide causes further losses $\left(L_{2}\right)$, resulting in the total detected output power $T$. At the same time the power reflected by the grating $\left(R_{\mathrm{G}} I_{\mathrm{G}}=\left(1-T_{\mathrm{G}}\right) I_{\mathrm{G}}\right)$, is back-coupled into a wire waveguide mode, resulting in the detected reflectance $R$.

Within this model, the reflection that is directly produced by the conversion of the wire waveguide mode into the grating Bloch mode (and vice-versa) is neglected at both interfaces. This assumption is quite reasonable for small filling factors when the shape discontinuity is smaller - and proves to be still valid for larger $f f$ (Palamaru and Lalanne 2001; Sauvan et al. 2005).

The following relationships can therefore be written:

$$
\left\{\begin{array}{l}
R+T+L=I \\
I_{\mathrm{G}}=I-L_{1} \\
T=T_{\mathrm{G}} I_{\mathrm{G}}-L_{2} \\
R=R_{\mathrm{G}} I_{\mathrm{G}}-L_{3} \\
L_{1}+L_{2}+L_{3}=L \\
L_{1}=\alpha I \\
L_{2}=\alpha T_{\mathrm{G}} I_{\mathrm{G}} \\
L_{3}=\alpha R_{\mathrm{G}} I_{\mathrm{G}}
\end{array}\right.
$$

where $\alpha$ is linked to the value of the overlap integral between the wire and grating Bloch mode (Palamaru and Lalanne 2001).

The equations can be solved and the transmittance and the reflectance of the isolated grating are found to be:

$$
\left\{\begin{array}{l}
T_{\mathrm{G}}=T /(R+T)=T /(I-L) \\
R_{\mathrm{G}}=R /(R+T)=R /(I-L)
\end{array}\right.
$$

\section{References}

Ahmad, R.U., F. Pizzuto, G.S. Camarda, R.L. Espinola, H. Rao and R.M. Osgood. IEEE Photonics Technol. Lett. 14 65, 2002.

Akahane, Y., T. Asano, B.S. Song and S. Noda. Opt. Expr. 13 1202, 2005. 
Almeida, V.R., C.A. Barrios, R.R. Panepucci and M. Lipson. Nature 431 1081, 2004.

Bellanca, G., P. Bassi, G. Erbacci, G. De Fabritiis and R. Roccari. Proceedings of the VII International

Conference "Microwave and High Frequency Heating", Valencia, 13-17 September 1999, 11, 1999.

Bienstman, P. and R. Baets. Opt. Quant. Electron. 33 327, 2001.

Chiang, K.S. IEEE Transactions on Microwave Theory and Techniques 44 692, 1996.

Čtyroký, J., S. Helfert, R. Pregla, P. Bienstman, R. Baets, R. De Ridder, R. Stoffer, G. Klaasse,

J. Petráček, P. Lalanne, J.P. Hugonin and R.M. De la Rue. Opt. Quant. Electron. 34 455, 2002.

Englund, D., I. Fushman and J. Vučković. Opt. Expr. 13 5961, 2005.

Espinola, R.L., R.U. Ahmad, F. Pizzuto, M.J. Steel and R.M. Osgood. Opt. Expr. 8 517, 2001.

Foresi, J.S., L.C. Kimerling, P.R. Villeneuve, S. Fan, J.D. Joannopoulos, E.P. Ippen, J. Ferrera, E.R.

Thoen, G. Steinmeyer and H.I. Smith. Nature 390 143, 1997.

Han, Y.T., J.U. Shin, D.J. Kim, S.H. Park, Y.J. Park and H.K. Sun. ETRI Journal 25 535, 2003.

Joannopoulos, J.D., R.D. Meade and R.N. Winn. Photonic Crystals: Molding the Flow of Light 1995.

Jugessur, A.S., P. Pottier and R.M. De la Rue. Opt. Expr. 12 1304, 2004.

Kimerling, L.C., L. Dal Negro, S. Saini, Y. Yi, D. Ahn, S. Akiyama, D. Cannon, J. Liu, J.G. Sandland,

D. Sparacin, J. Michel, K. Wada and M.R. Watts. Silicon Photonics 94 89, 2004.

Knox, R.M. and P.P. Toulios. Proceedings on Symposium on Submillimeter Waves, Brooklyn, 497, 1970.

Krauss, T.F., B. Vögele, C.R. Stanley and R.M. De la Rue. IEEE Photonics Technol. Lett. 9 176, 1997.

Lalanne, P. and J.P. Hugonin. IEEE J. Quantum Electron. 39 1430, 2003.

Noda, S., M. Imada, M. Okano, S. Ogawa, M. Mochizuki and A. Chutinan. IEEE J. Quantum Electron. $38726,2002$.

Palamaru, M. and P. Lalanne. Appl. Phys. Lett. 78 1466, 2001.

Palik, E.D. Handbook of optical constants of solids Academic Press, San Diego, 1998.

Sauvan, C., G. Lecamp, P. Lalanne and J.P. Hugonin. Opt. Expr. 13 245, 2005.

Taflove, A. Computational electrodynamics - The Finite Difference Time-Domain Method Artech House, Norwood, 1995.

Van Thourhout, D., P. Dumon, W. Bogaerts, G. Roelkens, D. Taillaert, G. Priem and R. Baets. Proceedings on 31st European Conference on Optical Communications (ECOC), Glasgow, 25-29 September 2005, 2, 241, 2005.

Zhao, C.Z., G.Z. Li, E.K. Liu, Y. Gao and X.D. Liu. Appl. Phys. Lett. 67 2448, 1995. 